\title{
ORIGINAL
}

PROF-1141

\section{PILONIDAL SINUS;}

USE OF LIMBERG FLAP IN THE TREATMENT

\author{
DR. FAISAL BILAL LODHI, FCPS \\ Assistant Professor \\ Allied Hospital Faisalabad \\ DR. SAIRA IJAZ, FCPS \\ Senior Registrar \\ Allied Hospital FAisalabad
}

\author{
DR. M. SHAFIQ, FCPS \\ Assistant Professor \\ Allied Hospital FAisalabad
}
Prof. Dr. Riaz Hussain, FRCS, FCPS
Surgical Unit-Il, Allied Hospital, Punjab Medical College, Faisalabad.

\begin{abstract}
Background: Pilonidal sinus is a common chronic disease of the sacrococcygeal region. The treatment for a pilonidal sinus varies according to the clinical presentation of the disease. Although many surgical methods have been suggested, an ideal method is still lacking because of high recurrence rates. Setting: Surgical Unit-II, Allied Hospital, PMC, Faisalabad. Material and Methods: This prospective study includes 30 patients who were treated with the use of a rhomboid excision and Limberg flap closure for chronic and recurrent sacrococcygeal pilonidal sinus. The follow-up period ranged from 04 to 08 months. Results: The mean hospital stay was 03 days (range, 03-08 days) and the mean time to return to work was 15 days (range, 12-26 days). Early wound complications were encountered in 03 patients. No recurrence was noted on maximum of 08 months of follow-up. Nine patients had numbness at the operation site and $10 \%$ were not pleased with cosmetic appearance of the scars. Conclusions: The results favor rhomboid excision and Limberg flap closure in the treatment of sacrococcygeal pilonidal sinus, especially in recurrent cases and in patients with extensive involvement. Low recurrence rates, shorter hospital stay, and time off from work may outweigh the disadvantages related to unfavorable cosmetic appearance.
\end{abstract}

Key words: Pilonidal sinus, Rhomboid excision, Limberg Flap

\section{INTRODUCTION}

Pilonidal disease was first reported in 1833. Sacrococcygeal pilonidal sinus is a common disorder among young adults affecting people aged 15-30 years. Pilonidal disease is now widely accepted as an acquired disorder.

The goals of the ideal procedure for the treatment of this disease include reliable wound healing with a low risk of recurrence, a short period of hospitalization, minimal inconvenience to the patient, and low morbidity with few wound-management problems. Treatment should allow the patient to resume normal daily activities as quickly as possible.

Skin flaps have been described to cover a sacral defect after wide excision. Similarly, this keeps the scar off the midline and flattens the natal cleft. The techniques 
available include the (1) cleft closure, (2) advancement flap (Karydakis procedure), (3) local advancement flap (3-plasty rhomboid flap or V-Y advancement flap), and (4) rotational flap (Limberg flap, Gluteus maximus myocutaneous flap).

\section{SETTING}

This study was conducted in Surgical Unit-II, Allied Hospital affiliated with Punjab Medical College, Faisalabad.

\section{METHODS}

This prospective descriptive study includes 30 patients, all males with age ranging between $25-42$ years. All patients were admitted through out patient department. Rhomboid excision and Limberg flap closure was done in all the patients under spinal anesthesia.

\section{PROCEDURE}

Spinal anesthesia was used in all the patient. 01gram of Third generation cephalosporin was given I/V at the start of the procedure. Initial marking (as shown in Fig 1) was done with patient in prone jack-knife position. The rhomboid $A B C D$ (having the pilonidal sinus) has all sides equal in length. Axis $A C$ is extended to $C H$. Axis $D C$ is extended to $\mathrm{CE}$. Axis $\mathrm{CF}$ is marked equidistant between Axis $\mathrm{CH}$ and $\mathrm{CE}$. Axis $\mathrm{FG}$ is marked parallel to Axis $\mathrm{BD}$. The length of both the Axes CF and $F G$ is equal to the length of the sides of rhomboid.

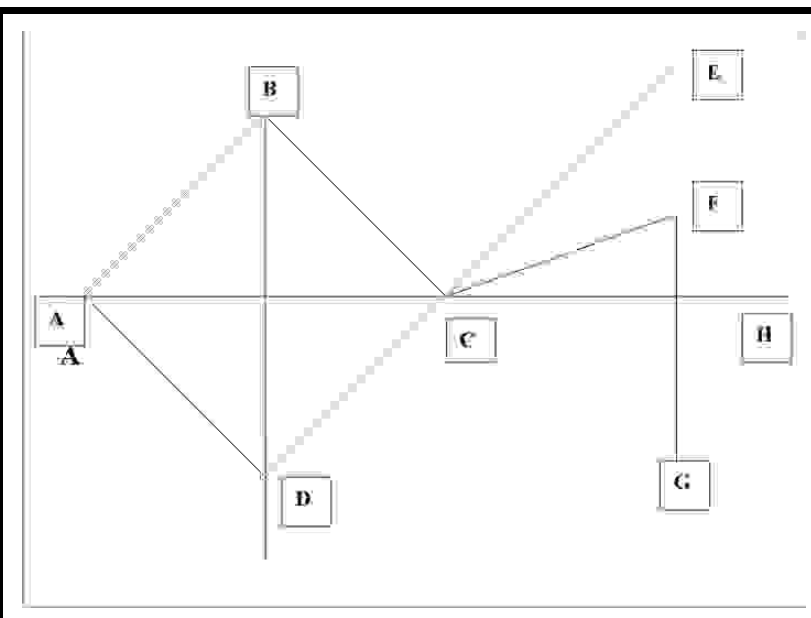

Fig-1.
Pilonidal sinus with all the side tracks was excised within the rhomboid ABCD (Fig 2, 3). The depth of the excision is upto the gluteal fascia. The flap was raised (Fig 4), with the depth upto gluteal fascia and rotated to cover the raw area (Fig 5). Meticulous haemostasis was ensured using electro-cautery and absorbable catgut sutures to prevent postop haematoma under the flap. Flap was stitched using deep stitches of Vicryl 2/0 and the skin stitched with Prolene 2/0. Suction drain was placed to prevent collection deep to the flap (Fig 6).

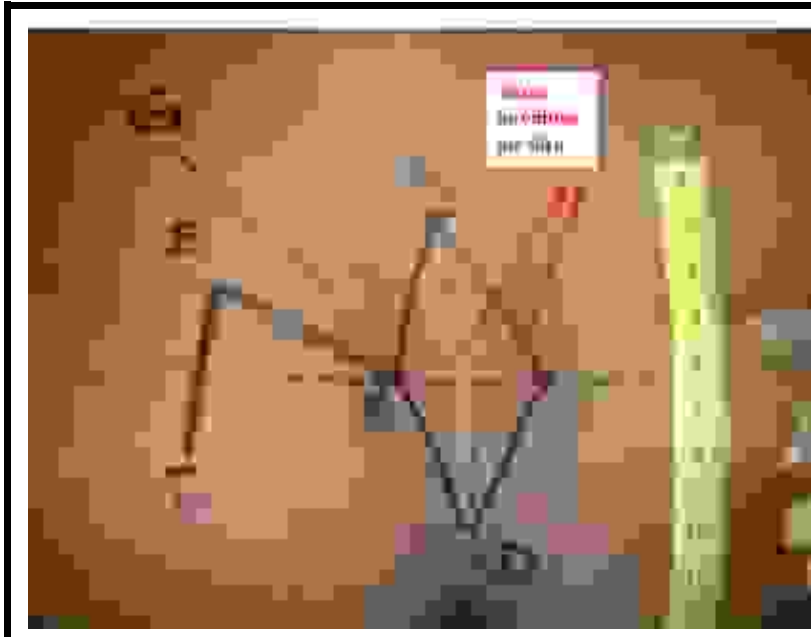

Fig-2.

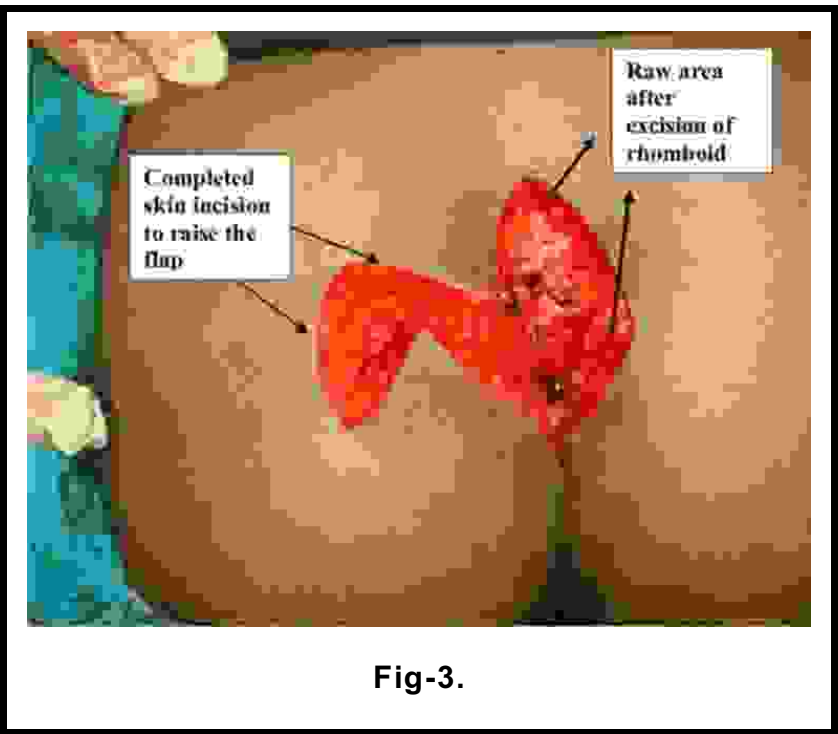




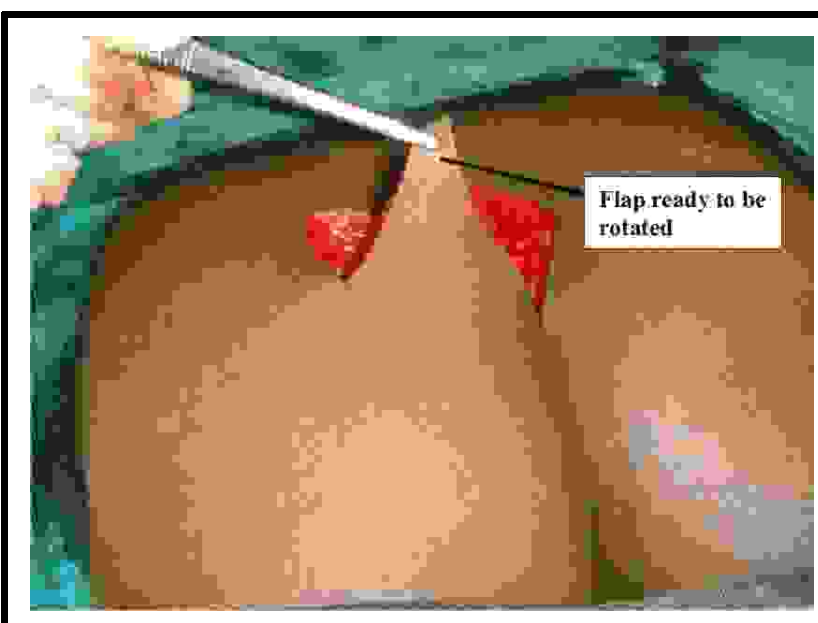

Fig-4.

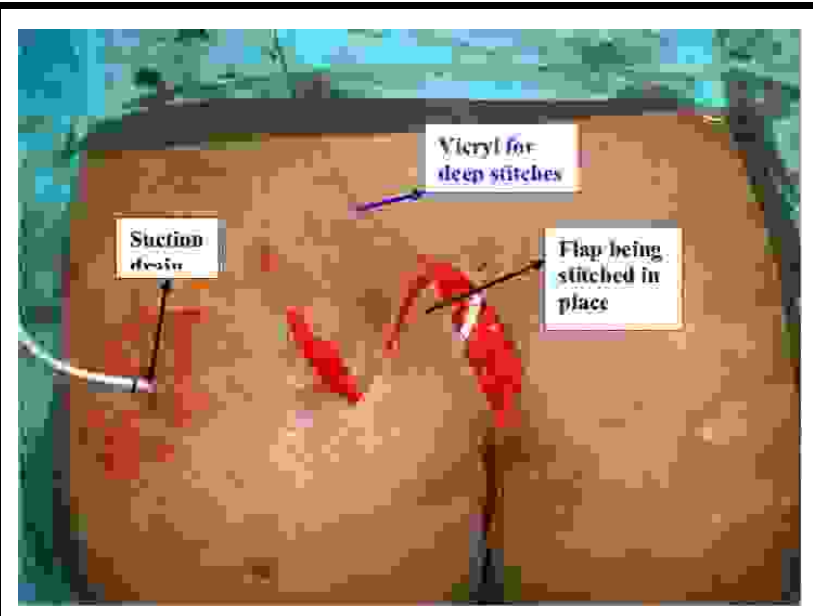

Fig-5.

Drain was removed at $3^{\text {rd }}$ to $4^{\text {th }}$ postop day. Skin stitches removed on $10^{\text {th }}$ postop day. Postoperative $01 \mathrm{gram}$ of Third generation cephalosporin was given $I / V$ twice daily for 02 days and then oral Amoxiclav combination was used for another 03 days. Patient advised to avoid pressure on the operated site for 2-3 days postoperatively.

\section{FOLLOW UP}

All the patients were advised to visit OPD on $10^{\text {th }}$ postop day for wound examination and removal of skin stitches. Then they were called at 4 weeks interval. Maximum follow up till this study is of 08 months. All the patients are advised to shave the area well around the operated site at least once fortnightly.

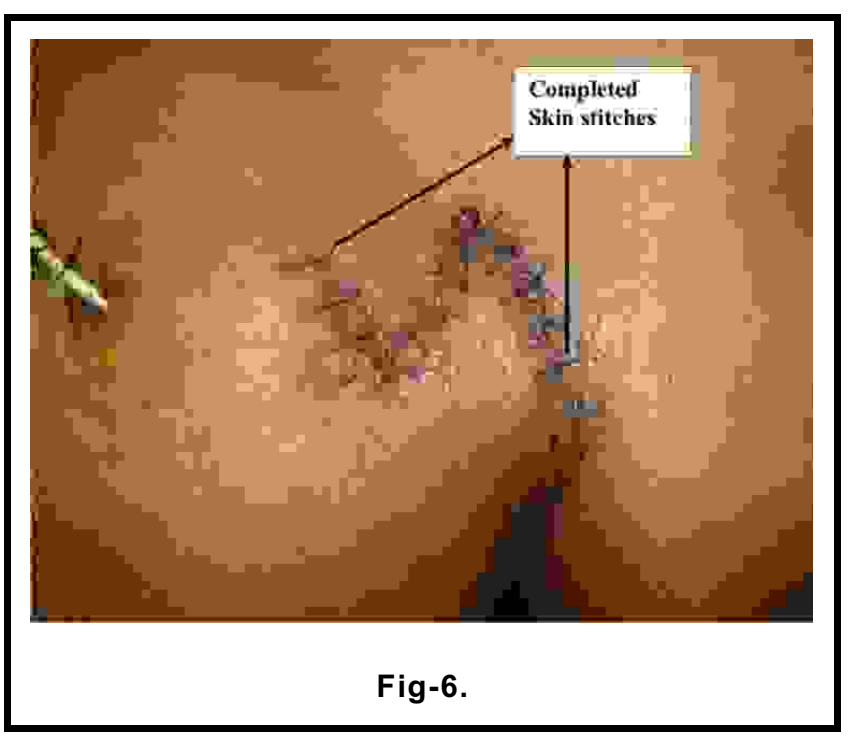

\section{RESULTS}

The mean hospital stay was 03 days (range, $03-08$ days). Two patients had to be hospitalized for 07-08 days due to superficial wound infection which recovered with injectable antibiotics.

The mean time to return to work after discharge from the hospital was 15 days (range, 12-26 days).

Early wound complications included superficial wound infection in 02 patients $(6.6 \%)$ and epidermal necrosis of flap edges in 01 patient (3.3\%).

Nine patients (30\%) had numbness at the operation site and three patients (10\%) were not pleased with cosmetic appearance of the scars. No patient has presented with recurrence on maximum of 08 months follow up.

\section{DISCUSSION}

Sacrococcygeal pilonidal disease is a common and well recognized entity. Malignant transformation is rare but cases of squamous cell carcinoma and verrucous carcinoma have been reported. Pilonidal sinus disease 
consists in a symptoms complex with presentations ranging from asymptomatic pits to painful draining lesions that are predominantly located in the sacrococcygeal region. Asymptomatic pits do not require treatment ${ }^{1}$. Many surgical procedures that are available for the symptomatic pilonidal sinus are well reviewed by AllenMersh in $1990^{2}$.

Ambulatory surgical treatments although effective but procedures which offer early recovery and early return to normal activity are the choice over complex procedures ${ }^{3 \text {, }}$ 4

The most difficult complication after the surgery for pilonidal sinus is persistently unhealed midline wound following lying open or excision of the primary disease ${ }^{5}$.

Although Karydakis has described a technique of asymmetrical closure with excellent results, this method is not popular ${ }^{6}$.

Many others including Mann and Springall have also described an asymmetrical excision and primary closure with good results using GA with a mean hospital stay of 16 days $^{7}$. Bascoms technique is popular method in West (Great Britain). It is done as a day care under local anaesthesia and sedation. Excision of midline pits and the abscess cavity is drained laterally $2-3 \mathrm{~cm}$ away from the midline and curetted. ${ }^{8}$

Buie first described an excision of pilonidal sinus and marsupilization in 1937 and published in $1944^{\circ}$. It was further advocated by Culp in $1967^{10}$. Although still used by some surgeons, this technique is associated with prolonged healing time and recurrence.

Wide local excision and primary closure has been advocated by some researchers because of its shorter healing time ${ }^{11}$ but the resulting scar remains in midline with increased incidence of recurrent disease.

For more complicated or recurrent diseases and nonhealing midline wounds more aggressive treatments such as split-thickness skin graft and Z-plasty are suggested. Skin grafts do not hold good at the area of friction and the Z-plasty has got results not so satisfactory to recommend its common use.

No treatment for pilonidal sinus is perfect. The reported rate of recurrence of pilonidal sinus varies widely in the literature from $0 \%$ to $43 \%{ }^{2}$.

Skin flaps have been described to cover a sacral defect after wide excision ${ }^{12}$. The Limberg flap technique involves a flap procedure to achieve primary closure and to obliterate the deep natal cleft. This relocates hair follicles away from the midline and prevents the frictional forces associated with the principal etiological factors for the development of pilonidal disease. Initially it was reserved for complex or recurrent pilonidal disease that has failed to respond to the simple conservative operative techniques but now it has been recommended as a first line management for all types of chronic sacrococcygeal pilonidal sinuses ${ }^{13,14}$. The potential complications include loss of skin sensation in the flap, which is observed in more than $50 \%$ of patients, and necrosis of the flap edges. Primary healing is achieved in $90 \%$ of cases ${ }^{15}$.

In summary, the goal for treatment of pilonidal disease is 2 -fold. The first is excising and healing with a low rate of recurrence. The second is minimizing patient inconvenience and morbidity after the surgical procedure and avoiding hospitalization with loss of workdays. The method used to treat the patient should satisfy these goals. Rhomboid excision and Limberg flap seems to be a satisfactory method in achieving a surgical cure of the disease ${ }^{14,15,16}$

\section{CONCLUSION}

The results favor rhomboid excision and Limberg flap closure in the treatment of sacrococcygeal pilonidal sinus. Low recurrence rates, shorter hospital stay, and time off from work may outweigh the disadvantages related to unfavorable cosmetic appearance.

\section{REFERENCES}

1. Chintapatla S, Safarani N, Kumar S, Haboubi N. Sacrococcygeal pilonidal sinus: historical review, pathological insight and surgical options. Curr Surg. 2005 Jan-Mar;40(2):80-84.

2. Allen-Mersh TG. Pilonidal sinus : finding the right tract 
for treatment. Br Surg 1990; $77: 123-32$.

3. Thompson Fawcett MW, Cook TA, Baigrie RJ, Mortensen NJMcC. What patients think of day-surgery proctology. BJ Surg 1998; $85: 1388$.

4. Royal College of Surgeons of England working party. Guidelines for day care surgery London. Royal College of Surgeons of England, 1992.

5. McLaren CA. Partial closure and other techniques in pilonidal surgery an assessment of 157 cases. $\mathrm{Br} \mathrm{J}$ Surg 1984; $71: 561-2$.

6. Karydakis GE. Easy and successful treatment of pilonidal sinus after explanation of its causative process. Aust NZJ Surg 1992; $62: 385-9$.

7. Mann CV, Springall RD. Excision for sacrococcygeal pilonidal sinus disease. Jr Soc Med 1987; 80 : 292-5.

8. Bascom J. Pilonidal Sinus. In current therapy in colon and rectal surgery. Toronto : BC Decker, 1990; 32-9.

9. Buie LA. Jeep disease. South Med J 1944; 37 : 103-9.

10. Culp CE. Pilonidal disease and its treatment Surg. Clin North America 1967; 47 : 1007-14.

11. Kronbag $\mathrm{O}$, Christiansen $\mathrm{K}$, Zimmermann-Nielson $\mathrm{C}$.
Chronic pilonidal sinus disease : A randomised trial with complete three year follow up. Br J Surg 1985; 72 :303-4.

12. Ertan $\mathrm{T}, \mathrm{Koc} M$, Gocmen $\mathrm{E}$ : Does technique alter quality of life after pilonidal sinus surgery? Am J Surg 2005 Sep; 190(3): 388-92.

13. Abu Galala KH, Salam IM, Abu Samaan KR, et al: Treatment of pilonidal sinus by primary closure with a transposed rhomboid flap compared with deep suturing: a prospective randomised clinical trial. Eur J Surg 1999 May; 165(5): 468-72.

14. Eryilmaz R, Sahin M, Alimoglu O, Dasiran F. Surgical treatment of sacrococcygeal pilonidal sinus with the Limberg transposition flap. Curr Surg. 2005 JulAug;62(4):387-90.

15. Urhan MK, Kucukel F, Topgul K, Ozer I, Sari S. Rhomboid excision and Limberg flap for managing pilonidal sinus: results of 102 cases. Diseases of the Colon Rectum 2002 May;45(5).

16. Mentes BB, Leventoglu S, Cihan A, Tatlicioglu E, Akin M, Oguz M. Modified limberg transposition flap for sacrococcygeal pilonidal sinus.Surgery Today, May 2004. 